\title{
Graphene and its Derivatives-Based Optical Sensors
}

\author{
Xiao-Guang Gao ${ }^{1,2}$, Ling-Xiao Cheng ${ }^{3 *}$, Wen-Shuai Jiang ${ }^{4 *}$, Xiao-Kuan $\mathrm{Li}^{2}$ and Fei Xing ${ }^{5 *}$ \\ ${ }^{1}$ College of Biomedical Engineering, Taiyuan University of Technology, Taiyuan, China, ${ }^{2}$ The Key Laboratory of Weak Light \\ Nonlinear Photonics, Ministry of Education, Nankai University, Tianjin, China, ${ }^{3}$ The First People's Hospital of Jinzhong, Jinzhong, \\ China, ${ }^{4}$ School of Biomedical Engineering, Xinxiang Medical University, Xinxiang, China, ${ }^{5}$ School of Physics and Optoelectronic \\ Engineering, Shandong University of Technology, Zibo, China
}

Being the first successfully prepared two-dimensional material, graphene has attracted extensive attention from researchers due to its excellent properties and extremely wide range of applications. In particular, graphene and its derivatives have displayed several ideal properties, including broadband light absorption, ability to quench fluorescence, excellent biocompatibility, and strong polarization-dependent effects, thus emerging as one of the most popular platforms for optical sensors. Graphene and its derivatives-based optical sensors have numerous advantages, such as high sensitivity, low-cost, fast

OPEN ACCESS

Edited by:

Karin Chumbimuni-Torres, University of Central Florida United States

Reviewed by: Gang Wei,

Qingdao University, China

Chih-Ching Huang,

National Taiwan Ocean University,

Taiwan

*Correspondence:

Ling-Xiao Cheng chenglingxiao01@163.com

Wen-Shuai Jiang jws19860826@126.com

Fei Xing

xingfei@sdut.edu.cn

Specialty section: This article was submitted to Analytical Chemistry, a section of the journal

Frontiers in Chemistry

Received: 08 October 2020 Accepted: 04 January 2021 Published: 05 February 2021

Citation:

Gao X-G, Cheng L-X, Jiang W-S, Li X-K and Xing F (2021) Graphene and its Derivatives-Based Optical Sensors.

Front. Chem. 9:615164.

doi: 10.3389/fchem.2021.615164 response time, and small dimensions. In this review, recent developments in graphene and its derivatives-based optical sensors are summarized, covering aspects related to fluorescence, graphene-based substrates for surface-enhanced Raman scattering (SERS), optical fiber biological sensors, and other kinds of graphene-based optical sensors. Various sensing applications, such as single-cell detection, cancer diagnosis, protein, and DNA sensing, are introduced and discussed systematically. Finally, a summary and roadmap of current and future trends are presented in order to provide a prospect for the development of graphene and its derivatives-based optical sensors.

Keywords: graphene, fluorescence, surface-enhanced Raman scattering, optical fiber, optical sensors

\section{INTRODUCTION}

The development of science often starts with unexpected experimental discoveries, which are even inconsistent with previous theories. However, it is through these unexpected discoveries that science has often advanced. For a long time in the past, scientists have believed that at room temperature, free-standing graphene could not exist due to the minimization of its surface energy (Peierls, 1935; Landau, 1937). This speculation was overthrown after the discovery of graphene in 2004 by Andre Geim and Konstantin Novoselov (Novoselov et al., 2004). Since it was first isolated via peeling graphite with adhesives, graphene has attracted tremendous attention from researchers due to its novel properties and wide range of applications (Gilje et al., 2007; Huang et al., 2011; Avouris and Dimitrakopoulos, 2012; Avouris and Xia, 2012; Yang et al., 2013; Gao et al., 2020). As firstly prepared two-dimensional materials, graphene is composed of a one atom-thick planar sheet of $s p^{2}$-bonded carbon atoms perfectly arranged in a honeycomb lattice (Novoselov and Geim, 2007; Nair et al., 2008; Neto et al., 2009). Graphene has many remarkable properties such as high mechanical strength (high Young's modulus of $1 \mathrm{TPa}$ ), thermal properties (high thermal conductivity $>3,000 \mathrm{WmK}^{-1}$ ), and excellent optical characteristics including broadband optical absorption in near infrared and visible range, and nonlinear optical properties, such as saturation absorption (Balandin et al., 2008; Bolotin et al., 2008; Bonaccorso et al., 2010; Koppens et al., 2011; Mak et al., 2012; Han et al., 2014). 
Due to its unique optical and electrical properties, graphene is widely used in photonic and optoelectronic devices, such as polarizers, modulators, ultrafast lasers, sensors, photodetectors and light-emitting diodes (Fowler et al., 2009; Cheng et al., 2010; Mueller et al., 2010; Sun et al., 2010a; Sun et al., 2010b; Wu et al., 2010; Bao et al., 2011; Kim and Choi, 2012; Liu et al., 2012; Sensale-Rodriguez et al., 2012; Son et al., 2012; Yuan and Shi, 2013; Liu et al., 2014).

By definition, an optical sensor is a device that can convert any external physical stimuli, such as electric field signals, pressure, heat, motion, sound, and biomolecules, into optical or electrical output for reading or further processing. For many years in the past, zero-dimensional material quantum dots, gold nanoparticles, one-dimensional material carbon nanotubes, and nanowires have been widely used in the design of optical sensors (Kong et al., 2001; Modi et al., 2003; Matsui et al., 2005; Frasco and Chaniotakis, 2009; Kuang et al., 2011; Shen et al., 2012). Since then, graphene has been widely employed in the field of optical sensors due to its unique properties. For example, graphene has the following characteristics: the thickness of one-atom layer, large specific surface area, high chemical stability, excellent biocompatibility, and the ability to absorb biomolecules through $\pi-\pi$ stacking. These properties allow for the fabrication of gas/chemical vapor, electromechanical, $\mathrm{pH}$, mass, electrochemical, and optical sensors. Graphene-based sensors have shown many advantages over conventional sensors, including reduced dimensions and weight, higher strength, ease of use and fabrication, and reduced cost. Furthermore, graphene derivatives have been also widely used in the field of optical sensors, including graphene oxide (GO), reduced graphene oxide (RGO), and graphene quantum dots (GQDs) (Robinson et al., 2008; Dong et al., 2010; Lipatov et al., 2013; Sun et al., 2013; Ananthanarayanan et al., 2014; Sansone et al., 2014; Wang et al., 2014; Wang et al., 2016). $\mathrm{GO}$ is not only an effective fluorescence quencher, but can also be used to achieve the detection of biomolecules with high sensitivity and high selectivity. Graphene prepared through mechanical exfoliation and chemical vapor deposition (CVD) methods has a high quality, but its low yield and high cost have seriously hindered its application. On the other hand, RGO is characterized by high yield and low cost, which render it more suitable for sensing application. In addition, compared with pure graphene, the oxygen functional group contained in RGO makes it easy to interact with biochemical molecules. At the same time, GQDs, another derivative of graphene, have also been extensively used in the field of biochemical sensing GQDs exhibit plenty of advantages such as excellent optoelectronic properties, extremely small size $(3-20 \mathrm{~nm})$, stable aqueous colloidal suspensions, ease of functionalization, and tunable fluorescence. Although there have been great achievements regarding graphene and its derivatives-based optical sensors, just a few reviews either highlights graphene-based fluorescence, or only mention graphene-based SERS. To better grasp the whole picture of this area, it is necessary to summarize the recent progress in graphene and its derivativesbased optical sensors. In this review, recent developments in

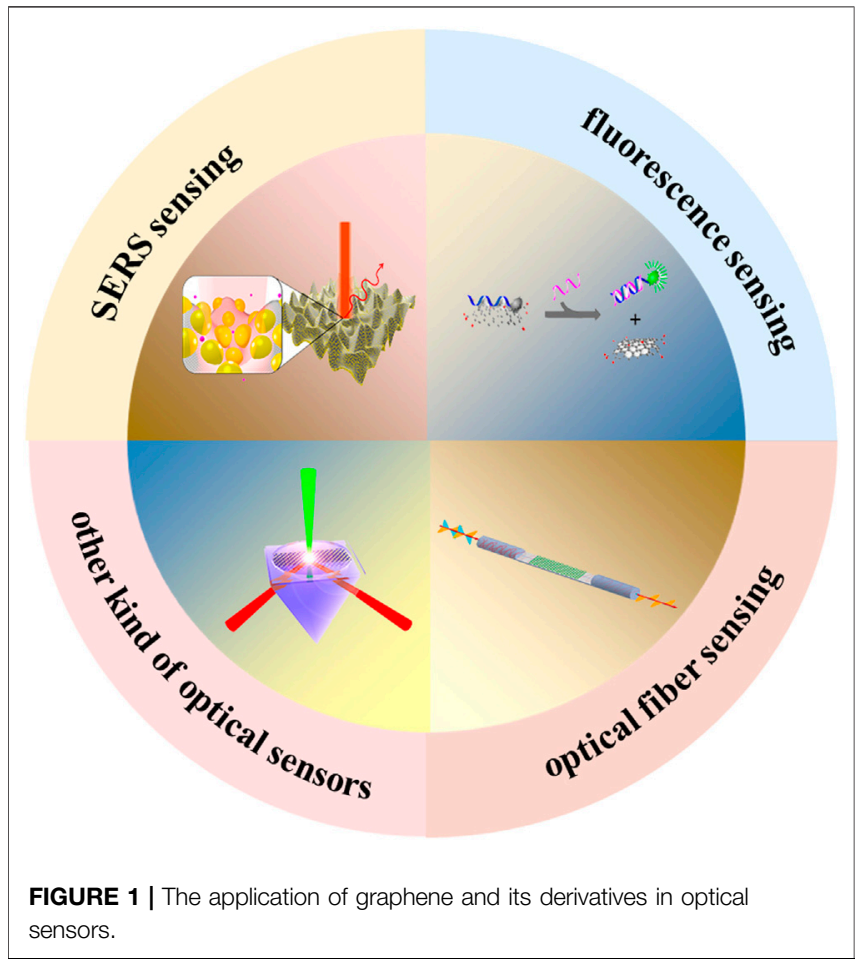

graphene and its derivatives-based optical sensors are summarized, covering aspects related to fluorescence, graphene-based substrates for surface-enhanced Raman scattering (SERS), optical fiber biological sensors, and other kinds of graphene-based optical sensors (Figure 1). Various sensing applications, such as single-cell detection, cancer diagnosis, protein, and DNA sensing, are introduced and discussed systematically. Finally, a summary on recent progress in graphene and its derivatives-based optical sensors is provided, alongside with a proposal for future applications and an outline for researches.

\section{FLUORESCENCE SENSING}

Graphene is composed of a one atom-thick planar sheet of $s p^{2}$ bonded carbon atoms, which exhibits no fluorescence characteristics due to its zero band gap (Novoselov et al., 2012; Zheng and $\mathrm{Wu}, 2017)$. The rich oxygen-containing functional groups contained in GO and RGO not only make them to be composed of $s p^{2}$ and $s p^{3}$ carbon atoms, but also give them a band gap and result in fluorescence (Huang et al., 2012; Mathkar et al., 2012). Compared with traditional fluorescent materials, GO has the characteristics of low cost, nontoxic, biocompatible, and environmentally friendly. In addition, GO can achieve adsorption of biomolecules through electrostatic force, hydrogen bonding or $\pi-\pi$ interactions, which also provides conditions for the interaction between biomolecules. Therefore, GO has been widely used in fluorescence sensing (Eda and Chhowalla, 2010; Loh et al., 2010; Zhu et al., 2010; Dreyer et al., 2011; Qiang et al., 2014). In this section, the principle and 

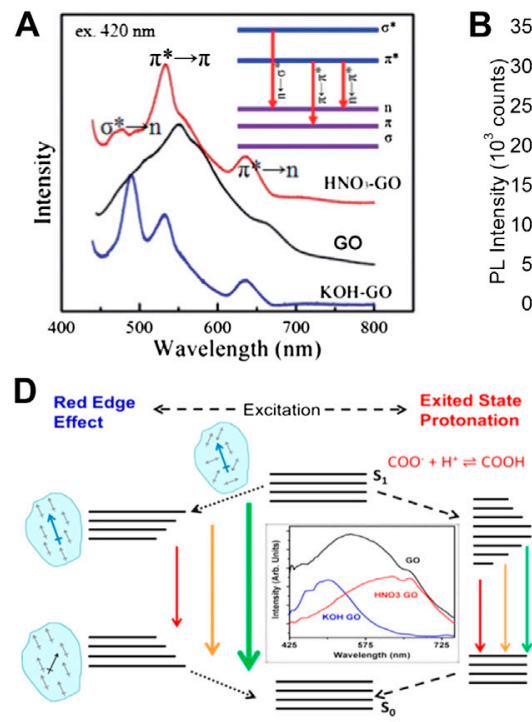

$\mathbf{G}$

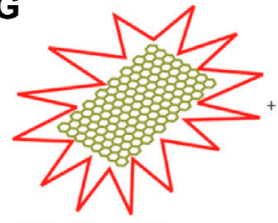

GO: Strong fluorescenc

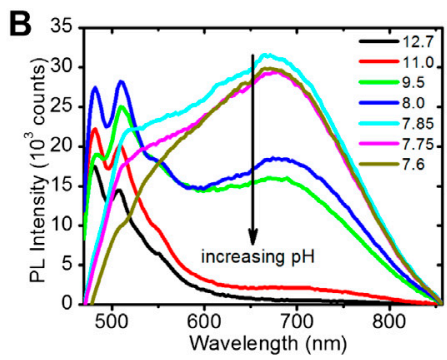

E
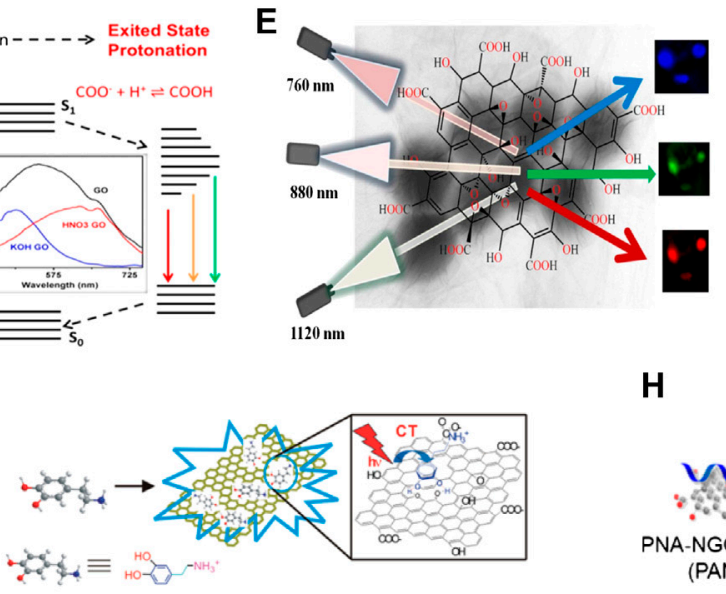

GO/DA complex: Weak fluorescence

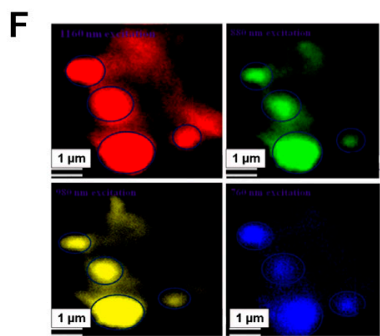

H
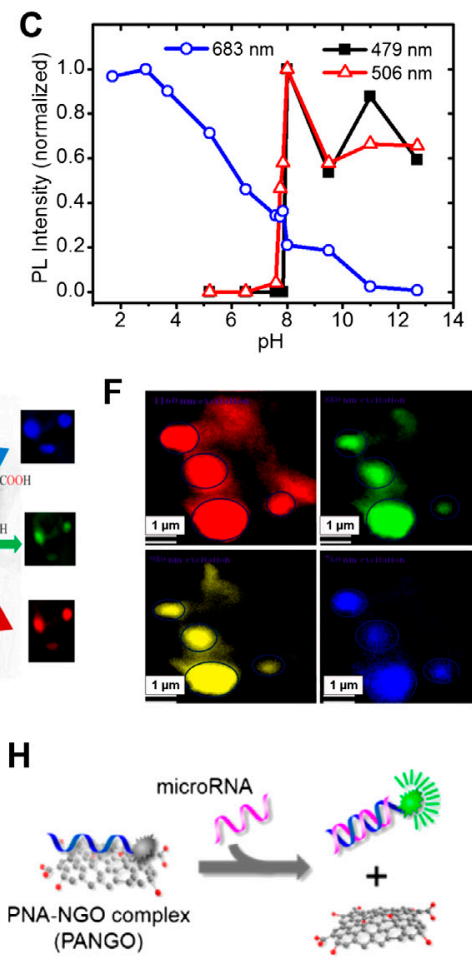

FIGURE 2 | The application of graphene and its derivatives in fluorescence sensing. (A) Fluorescence spectra corresponding to GO and GO treated with KOH and $\mathrm{HNO}_{3}$; inset: the different electronic transitions (Li et al., 2012). (B) The pH-dependent photoluminescence of GO. (C) The intensities of photoluminescence at different wavelengths $(\lambda=683,506$, and $479 \mathrm{~nm}$ ) and its corresponding pH value (Galande et al., 2011). (D) Fluorescence mechanism of GO (Cushing et al., 2014). (E) Tunable excitation-wavelength-dependent two-photon imaging. (F) Two-photon fluorescence imaging technique based on aptamer-modified GO and its application (Pramanik et al., 2014b). (G) High-sensitivity detection of dopamine by using GO as a fluorescence quencher (Chen et al., 2011). (H) MicroRNA (miRNA) detection based on GO and peptide nucleic acid (PNA) (Ryoo et al., 2013).

properties of GO fluorescence will be introduced in detail. The application of GO fluorescence in the field of sensing will then be discussed.

The fluorescence of GO comes from its electronic energy transitions. As displayed in Figure 2A, each fluorescence peak of functionalized GO is derived from the corresponding specific electronic transitions (Zhu et al., 2011). In addition, GO contains various types of oxygen-containing functional groups, such as epoxy (C-O-C), carboxyl groups $(\mathrm{COOH})$, hydroxyl groups $(\mathrm{COH})$, and aromatic rings $(\mathrm{C}=\mathrm{C})$, which lead to the overlap of many fluorescence peaks. The position and intensity of the fluorescence peak of GO is highly susceptible to functional groups, solvents, localized domains, and strain. As shown in Figure 2A, the fluorescence peak position and intensity are modified when $\mathrm{GO}$ is enriched with $\mathrm{OH}$ or $\mathrm{COOH}$ groups, respectively (Li et al., 2012). Figures 2B,C show the effect of $\mathrm{pH}$ on the fluorescence of GO (Galande et al., 2011). Under acidic conditions, GO exhibits a broad fluorescence peak centered at $\sim 680 \mathrm{~nm}$. However, as the $\mathrm{pH}$ value increases, an amazing phenomenon could be observed. One fluorescence peak $(\sim 680 \mathrm{~nm})$ gradually decreases until it disappears, and another fluorescence peak $(\sim 500 \mathrm{~nm})$ appears under basic conditions. This change in the fluorescence properties caused by $\mathrm{PH}$ is mainly due to the excited-state proton transfer, as shown in Figure 2D (Cushing et al., 2014). Under acidic conditions, the $\mathrm{COOH}$ group mainly exists in the aqueous solution in the form of ionic $\mathrm{COO}^{-}$. Under excitation of incident bean, the two kinds of ions $\mathrm{COO}^{-}$ and $\mathrm{COOH}$ in the excited-state contributes to the broad fluorescence peak at $668 \mathrm{~nm}$. Furthermore, the fluorescence of GO displays excitation-wavelength-dependent properties due to the giant red-edge effect. As displayed in the Figure 2D, due to the extra relaxation process introduced by the polar solvent, such as water, a giant red-edge effect can be observed When the solvation dynamics occur on a timescale which is orders of magnitude shorter than that of the fluorescence, the solvation is usually completed prior to fluorescence. Therefore, the final fluorescence only undergoes a small redshift.

In fluorescence sensing, when GO is used as a fluorescent chromophore, its fluorescent properties can be modulated by changing its sheet size, chemical composition and other factors. Then, through the process of resonance-energy-transfer and carrier transport, GO is often used as a fluorescence quencher in the field of fluorescence sensing. This dual role of GO as both a fluorophore and a quencher have also brought new ideas to sensor design. Although GO could in principle be used as a fluorescent label, its broad peak limits its sensing performance. 
Instead, GO is remarkably suitable for near infrared (NIR) biological imaging via two-photon excitation spectroscopy thanks to the giant red-edge effect (So et al., 2000; Tsai et al., 2013; Pramanik et al., 2014b). In 2014, Pramanik et al. developed graphene oxide sheet based two-photon multi-color bio-imaging of multiple drug-resistance bacteria (MDRB), where multicolor imaging is based on the fact that the two-photon fluorescence wavelength of the graphene oxide sheet can be tuned just by varying the excitation energy without changing its chemical composition and size (Galande et al., 2011). As displayed in Figure 2E, the imaging color and luminescence peak position can be tuned from deep blue to red, just by varying the excitation wavelength. Figure 2F displays multicolor two-photon luminescence imaging of methicillin-resistant Staphylococcus aureus (MRSA) at different wavelengths excitation. Furthermore, two photon imaging with GO as a fluorescent label has been widely used in the fields of cancer cell imaging and food safety (Pramanik et al., 2014a; Shi et al., 2015; Tchounwou et al., 2015; Wang et al., 2015b). In 2016, Kalluru et al discovered that modified nano-sized GO exhibits wavelength of the excitation dependent fluorescence effect, which is suitable for fluorescence imaging (Kalluru et al., 2016). In addition, the functionalized nano-sized GO can generate a large amount of singlet oxygen through the irradiation of near-infrared light, which is used for photodynamic therapy and photothermal therapy The consequence of experiments also proved that photodynamic and photothermal treatment based on functionalized nano-sized GO can effectively extend the lifespan of mice. In 2019, Song et al. creatively encapsulated nanocrystals doped with rhenium into GO (NCs@GO) (Song et al., 2019). The NCs@GO not only has a strong fluorescence effect in the visible and near infrared bands, but also has satisfactory solubility and biocompatibility. Then the NCs@GO has been utilized not only to achieve high-sensitivity detection of miRNA in cells, but also to realize real-time imaging of tumors.

$\mathrm{GO}$ as an effective fluorescence quencher is also widely used in fluorescence sensing. Figure 2G displays the GO being used as an efficient quencher through charge-transfer (Chen et al., 2011). Only when the distance between the donor and the acceptor of the charge is less than $10 \AA$, the charge transfer process is possible. Such a short distance is often obtained through chemical bonding or physical adsorption. In 2011, Chen et al. a GO-based photoinduced charge transfer (PCT) label-free near-infrared (near-IR) fluorescent biosensor for dopamine. The multiple non-covalent interactions between GO and dopamine can achieve effective fluorescence quenching and high-sensitivity detection of dopamine. In 2013, Wu et al. used GO as a fluorescence quencher to detect heavy metal cations ( $\mathrm{Li}$ et al., 2013a). The principle involved in the experiment is displays as follows: first, the aptamer must be modified on the surface of GO. When the aptamer captures $\mathrm{Hg}^{2+}$, a hairpin structure is formed. In this structure, the charge transfer process between GO and mercury ions causes the fluorescence of GO to be quenched. The present optical sensor shows a limit of detection as low as $0.92 \mathrm{nM}$ and excellent selectivity over various metal ions. The fluorescence sensing based on charge transfer has been applied to the detection of more types of pollutants in environment and agriculture (Zhu et al., 2014; Wang et al., 2015a; He et al., 2015). Fluorescence resonance energy transfer (FRET) is a nonradiative energytransfer process based on the dipole-dipole interaction (Jung et al., 2010; Kwak et al., 2014). It requires the donor's emission spectrum to overlap with the acceptor's absorption spectrum. In the FRET process, GO can serve as an energy donor in which its fluorescence gets quenched by an energy acceptor, such as gold nanoparticles or organic dyes. Furthermore, GO can also act as an energy acceptor, in which it quenches the fluorescence of an energy donor, such as quantum dots and organic dyes. In 2013, Ryoo et al. developed a GO based miRNA sensor, which allows quantitative monitoring of target miRNA expression levels in living cells (Ryoo et al., 2013). The strategy is based on tight binding of GO with peptide nucleic acid (PNA) probes, resulting in fluorescence quenching of the dye that is conjugated to the PNA, and subsequent recovery of the fluorescence upon addition of the target miRNA. The present miRNA sensor allowed the detection of specific target miRNAs with a detection limit as low as $\sim 1 \mathrm{pM}$, as well as the simultaneous monitoring of three different miRNAs in a living cell. By inoculating different aptamers and antibodies on the surface of $\mathrm{GO}$, GO-based detection platforms will be used in more fields ( $\mathrm{He}$ and Cui, 2012; Al-Ogaidi et al., 2014; Iranifam et al., 2016). Compared with traditional organic dye molecules and other types of nanomaterials, graphene and its derivatives have the advantages of photostability and biocompatibility. In addition, graphene and its derivatives could be used not only as fluorophore, but also as fluorescence quenchers in fluorescence sensing, making it widely used in the fields of biosensing and bioimaging. However, the oxygen-containing functional groups in GO form a broad fluorescence peak, which limits its application in biosensing. Therefore, the functional treatment of GO is very critical.

\section{Graphene-Based Surface-Enhanced Raman Scattering Sensing}

Due to the extremely high detection sensitivity and the capability of chemical fingerprints recognition, SERS has been an attractive analytical technique used in various fields (Kneipp et al., 1997; Li et al., 2011; Alvarez-Puebla and Liz-Marzán, 2012; He and Cui, 2012; Zhang et al., 2013). Furthermore, since the phenomenon of graphene based surface enhanced Raman scattering was discovered, the graphene based substrate has attracted wide attention of researchers. Compared with traditional Raman substrates, the introduction of graphene has many advantages, and at the same time it has brought tremendous development. Graphene can not only effectively quench the photoluminescence of fluorescent dyes and drastically eliminate the fluorescence background, but it can also cooperate with typical noble metallic nanoparticles as high-performance SERS substrates. Furthermore, it can serve as an excellent charge transfer-only SERS substrate for understanding the exact role of chemical mechanism without the interference of electromagnetic mechanism (Dalfovo et al., 2014; Lin et al., 2017). In this section, graphene-based SERS substrates and the role played by graphene in graphene-based SERS substrates are 

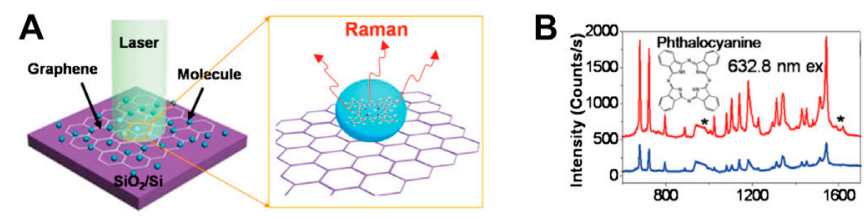

D
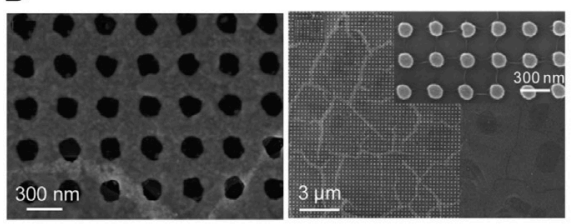

$E$

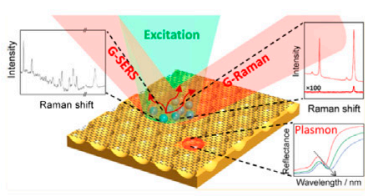

G

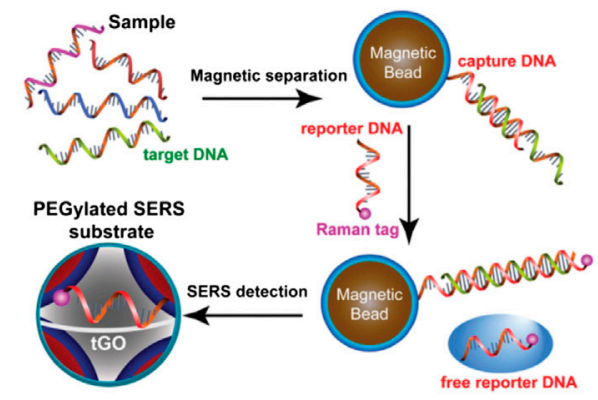

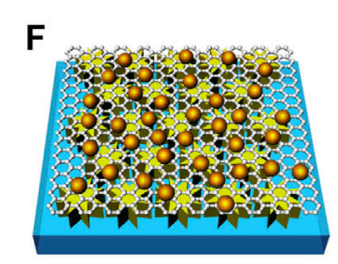
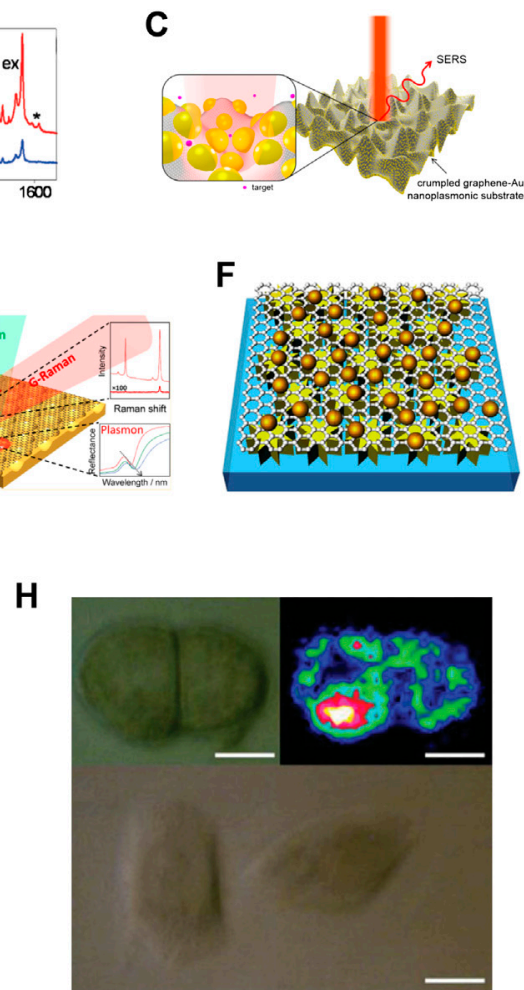

FIGURE 3 | The graphene-based substrates for surface-enhanced Raman scattering (SERS). (A) Schematic illustration of the graphene only substrates. (B) Raman spectra of Pc molecules on monolayer graphene and on a blank $\mathrm{SiO}_{2} / \mathrm{Si}$ substrate (Ling et al., 2010). (C) Schematic of SERS enhancement from a crumpled graphene-Au nanoparticles hybrid structure (Leem et al., 2015). (D) Graphene-covered nanoparticles or nanohole arrays for SERS enhancement (Hao et al., 2012). (E) Graphene-covered Au nanovoid arrays (Zhu et al., 2013). (F) Graphene-separated metal nanostructure substrates (Zhao et al., 2017). (G) Schematic illustration of the SERS detection of DNA based on graphene (Duan et al., 2015). (H) Optical and Raman images of HeLa 229 cells. The scale bar is 10 $\mu$ m (Zhang et al., 2015 ).

summarized. Furthermore, the SERS applications of graphenebased substrates in biomedical areas, including biomolecule detection and bio-imaging are discussed.

Graphene exhibits universal absorption independent of wavelength in the visible range, and has plasmon resonance in the terahertz. The Raman enhancement effect of graphene is mainly attributed to the charge transfer between graphene and various molecules, which result in a chemical enhancement. In 2010, Ling et al. explored the possibility that graphene could be used as a substrate for enhancing Raman signals of adsorbed molecules (Lai et al., 2018). Several different types of molecules, used as Raman probe, were deposited through an identical process both on graphene and on a $\mathrm{SiO}_{2} / \mathrm{Si}$ substrate using vacuum evaporation or solution soaking. By comparing the Raman signals of molecules on graphene and on a $\mathrm{SiO}_{2} / \mathrm{Si}$ substrate, they demonstrated that the intensities of the Raman signals on graphene were much stronger than on a $\mathrm{SiO}_{2} / \mathrm{Si}$ substrate. Figure 3A displays the graphene-only substrates. In Figure 3B, the intensities of the Raman signals of phthalocyanine (Pc) on graphene are much stronger indicating that graphene can enhance the Raman signals of these molecules.

In addition, graphene derivatives-based substrates have also been extensively studied. In 2013, Liu et al. explored the effects of $\mathrm{pH}$ values on SERS intensities of some aromatic molecules on
GO. They concluded that the GO-mediated SERS is associated with charge-type selectivity according to electrostatic interactions (Liu et al., 2013). In 2016, Yin et al. explored the SERS effects of RGO with different degrees of reduction (Yin et al., 2016). They found that the Raman intensities of RGO decreased with increasing the reduction duration from 2 to $60 \mathrm{~min}$, while the strongest Raman intensity of R6G molecules was observed for $10 \mathrm{~min}$. Graphene-only substrates provide a better way to understand the chemical mechanism of SERS, but their enhancement effect remains still no strong enough for application. Therefore, it is necessary to develop graphene-metal substrates for highly sensitive SERS analysis. Figure 3C displays the graphene-supported nanostructures substrate. Leem et al. developed a mechanical self-assembly strategy to enable a new class of 3D crumpled graphene-gold $\mathrm{Au}$ ) nanoparticles hybrid nanoplasmonic structures for SERS applications (Leem et al., 2015). The 3D crumpled graphene-Au NPs exhibit at least one order of magnitude higher SERS detection sensitivity than that of conventional, flat graphene$\mathrm{Au}$ NPs. The hybrid structure is further adapted to arbitrary curvilinear structures for advanced, in situ, nonconventional, nanoplasmonic sensing applications. The graphene-covered metal nanostructures substrates are displayed in Figures 3D,E (Hao et al., 2012; Zhu et al., 2013). In this structure, graphene can 
serve not only as a protective layer, so that the metal microstructure is not oxidized and corroded, but also to avoid direct contact between the biomolecules and the metal microstructure, thus allowing to exclude the side photocatalytic reaction. The graphene-separated metal nanostructure substrate is displayed in Figure 3F. Zhao et al. developed an efficient SERS substrate by sandwiching graphene between Au NPs and electron beam lithography-fabricated Ag nanostar arrays (NSAs). The fabricated hybrid structure exhibits 137-fold enhancement of the Raman response of graphene, with a limit of detection of $0.1 \mathrm{pM}$ for rhodamine $6 \mathrm{G}$ molecules (Zhao et al., 2017).

Undoubtedly, graphene has been widely employed in the design of SERS substrates. Graphene has overcome some of the limitations of the SERS substrates since it endows the SERS substrates with better stability, sensitivity, reproducibility, and biocompatibility. More importantly, highperformance graphene based substrates have great application potential in numerous fields. Various biomarkers and biomolecules, such as DNA, nucleosides, proteins, bacteria, and fungi have been successfully detected through the employment of multiple graphene-based substrates (Kang et al., 2010; Li et al., 2013b; Lin et al., 2014; Duan et al., 2015; Xu et al., 2015; Ouyang et al., 2017). In addition, graphene-based substrates have been shown to be effective for bio-imaging, cancer diagnostics, drug delivery, photothermal therapy and chemotherapy (Ilkhani et al., 2016; Cialla-May et al., 2017). Figure 3G displays an example of DNA detection using graphene-based SERS sensing. In 2015, Duan et al. developed a new type of SERS substrate with thiolated graphene oxide (tGO) nanosheets sandwiched between two layers of closely packed plasmonic nanoparticles (Duan et al., 2015). Herein, tGO can play multifunctional roles as a $2 \mathrm{D}$ scaffold to immobilize interfacially assembled plasmonic nanoparticles, a nanospacer to create SERS-active nanogaps between two layers of nanoparticle arrays, and a molecule harvester to enrich molecules of interest via $\pi-\pi$ interaction. Furthermore, they demonstrated that an SERS assay based on the PEGylated substrate, in combination with magnetic separation, allows for sensitive, multiplexed "signal-off" detection of DNA sequences of bacterial pathogens. Cell imaging is a powerful method to reveal mechanisms and cellular processes and to diagnose diseases. In Figure 3H, Zhang et al. developed the Raman-fluorescence dual imaging of cells based on a multifunctional GO/AuNPs/2aminoethanethiol (AET)/fluorescein isothiocyanate hybrid platform (Zhang et al., 2015). Raman imaging of HeLa 229 cells with GO/AuNPs/p-ATP hybrids showed excellent performance as the characteristic peaks of p-ATP at 1,078 , $1,137,1,330,1,435$, and $1,590 \mathrm{~cm}^{-1}$ could be clearly observed. In 2017, Zhang et al. prepared Au triangular nanoarray/ graphene/Au nanoparticles sandwich structure as the SERS substrate (Zhang et al., 2017). They have proved that the hightemperature annealing process can effectively reduce the distance between the $\mathrm{Au}$ nanoparticles and the Au triangular nanoarray, and achieve effective amplification of the Raman signal. Then the excellent SERS platform has been used for high sensitivity detection of mercury ions $\left(8.3^{\star} 10^{-9} \mathrm{M}\right)$. In 2018 , Zeng et al. successfully synthesized silver nanospheres coated with graphene oxide (Ag@NGO) (Zeng et al., 2018). The hybrid nanomaterials not only can effectively enhance the Raman signal, but also its stability is greatly improved due to the presence of GO. Then they proved that Ag@NGO nanoparticles can not only be used as an effective nano-probe to monitor intracellular biological molecular, but also can serve as a drug delivery nano-carriers as well by $\pi-\pi$ interaction with anticancer drug DOX. In 2020, Choi et al. synthesized GO-coated gold nanoarrays in the shape of tooth to achieve effective Raman signal enhancement (Choi et al., 2020). Then the GO hybrid structure were utilized to achieve rapid and highly sensitive detection of dopamine $\left(10^{-4}\right.$ to $\left.10^{-9} \mathrm{M}\right)$. In additional, SERS based on a reliable graphenebased substrate has been successfully used to discriminate various environment pollutants, including organic pollutants, heavy metal ions, pathogens, and antibiotics. Compared with traditional SERS substrates, graphene has proper modification and improved biocompatibility, which also makes graphenebased SERS substrates widely used in biosensing, drug delivery, and bioimaging fields. However, graphene-based substrates also need to address many difficulties and challenges. First of all, the stability of graphene-based substrates needs to be improved, especially when various biochemical reactions occur on the surface of graphene. In addition, non-specific adsorption on the graphene surface will also cause serious disturbances. Therefore, it is urgent to develop graphene based substrates with high stability and high specificity.

\section{Graphene-Based Optical Fiber Sensing}

Optical fiber sensors have received world-wide attention due to their high sensitivity, small size, good anti-electromagnetism disturbance ability and other potential advantages. As the first prepared two-dimensional material, graphene has been used extensively in the design of optical fiber sensors due to its unique optical properties (Yao et al., 2014a; Zhang et al., 2014; Dash and Jha, 2015; Li et al., 2015; Zeng et al., 2015). In this section, the principles of different types of graphene-based optical fiber sensors and their applications in biochemical sensing are introduced in detail. It was shown that graphene had a great potential in the optical fiber sensing technology.

Graphene based fiber optical sensors with various device configurations for biochemical sensing are displayed in Figure 4. Figure $4 \mathrm{~A}$ shows a schematic structure of a thin layer of graphene wrapped around a sub-wavelength-diameter $(1 \mu \mathrm{m})$ tapered single-mode microfiber (Shivananju et al., 2017). In this structure, tapered or micro fibers are usually prepared via a chemical etching process or a gently stretching process while heating over a flame or using a heated filament. During the preparation process, the fiber core becomes thinner, causing the evanescent wave to reach the outside and thus being exposed to the surrounding medium through the graphene. A surface plasmon resonance (SPR) sensor is obtained by placing a graphene layer over the tapered region, and is widely used in gas sensing. In 2014, Yao et al. developed an all-optical $\mathrm{NH}_{3}$ gas sensor based on graphene/microfiber hybrid waveguide (GMHW) (Yao et al., 2014a). During the fabrication process of the GMHW sensor, they transferred the graphene prepared via 


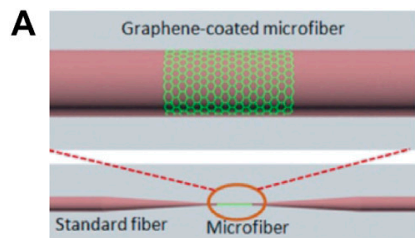

B

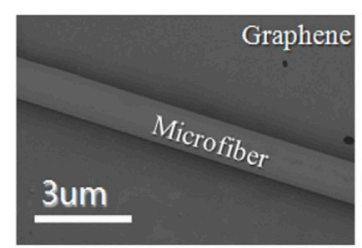

C

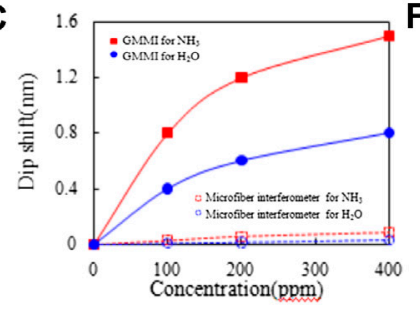

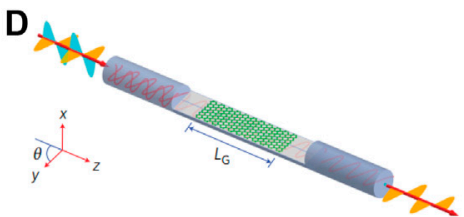

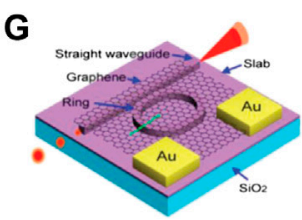

E

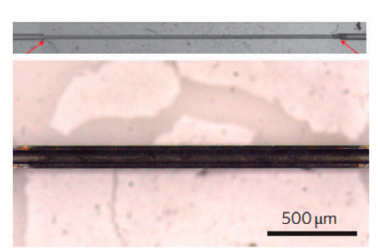

$\mathbf{F}$

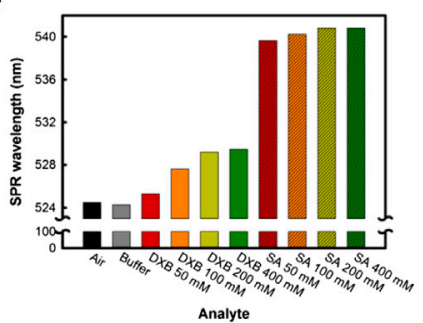

H

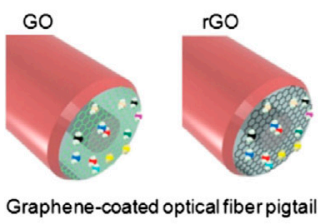

I

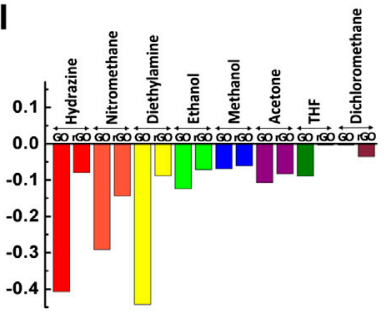

FIGURE 4 | The graphene based fiber optical sensors. (A) Schematic diagram of the graphene/microfiber hybrid waveguide (Shivananju et al., 2017). (B) Scanning electron microscopy (SEM) image of the graphene-coated microfiber waveguide (Yao et al., 2014a). (C) Graphene-coated microfiber waveguide used for high-sensitivity gas sensing (Yao et al., 2014b). (D) Schematic model of the fiber-to-graphene coupler based on a side-polished optical fibre. (E) Optical images of a laterally polished optical fiber and of a planar section of the optical fiber covered by few-layer graphene (Bao et al., 2011). (F) The fiber-to-graphene coupler were used for highsensitivity protein sensing (Kim et al., 2013). (G) Illustration of graphene-coated optical microring resonator (Gan et al., 2015). (H) Schematic of the end of an optical fiber pigtail with a graphene coating on pinhole (Shivananju et al., 2017). (I) Comparative plots of the sensing responses of GO and RGO-coated polymer optical fibers (Some et al., 2013).

CVD to the $\mathrm{MgF}_{2}$ substrate. The graphene/ $\mathrm{MgF}_{2}$ substrate was then fixed on a translation stage and the microfiber was attached onto the graphene. The SEM image of the graphene-coated tapered microfiber is depicted in Figure 4B. The adsorption of $\mathrm{NH}_{3}$ can change the effective refractive index of the GMHW. The wavelength shift induced by the $\mathrm{NH}_{3}$ absorption is spectrally demodulated using a microfiber-based Mach-Zehnder interferometer. The GMHW has been demonstrated to have a high sensitivity of $\sim 6 \mathrm{pm} / \mathrm{ppm}$, and a resolution of $0.3 \mathrm{ppm}$. In 2014, Yao et al. observed the enhancement of the surface evanescent field by graphene cylindrical cladding (Yao et al., 2014b). It was found that the light in the fiber core can be effectively modulated by graphene, improving the detection sensitivity of the hybrid waveguide. The experimental results for gas sensing verified the theoretical prediction, and ultra-high sensitivities of $\sim 0.1 \mathrm{ppm}$ for $\mathrm{NH}_{3}$ gas detection and $\sim 0.2 \mathrm{ppm}$ for $\mathrm{H}_{2} \mathrm{O}$ vapor detection were achieved (Figure 4C).

Figure 4D displays a schematic diagram of a fiber-to-graphene coupler based on a side-polished or D-shaped optical fiber (Bao et al., 2011). The optical images of a laterally polished optical fiber and of a planar section of optical fiber covered by few-layer graphene are displayed in Figure 4E. The working principle of this structure is based on the interaction of the evanescent wave with the surrounding environment. In 2014, Kim et al. developed graphene-based D-shaped fiber optic SPR for biochemical sensing applications (Kim et al., 2013). A graphene film synthesized via thermal chemical vapor deposition is transferred onto the sensing area of the optical fiber. The detection mechanism of this sensor is based on the principle that the SPR signal changes according to the refractive indices of the analytes. In this experiment, the LED light $(524 \mathrm{~nm})$ is polarized and coupled into the graphene-coated fiber sensor. The change in the polarized light due to the change in the refractive index, caused by biomolecule interaction, is captured by spectrometer. Figure 4F displays the bar chart for SPR wavelengths for each analyte. The SPR peaks appear at $\sim 524 \mathrm{~nm}$ for the buffer case and the case without analyte, $525.3-529.5 \mathrm{~nm}$ for biotinylated double crossover DNA (DXB) samples, and 539.6-540.8 nm for protein streptavidin (SA) samples. The consequences of this experiment verify the sensitivity and selectivity of graphenecoated fiber sensors for protein and DNA detection. Figure 4G displays a schematic diagram of the optical ring resonator covered with graphene (Gan et al., 2015). The graphene-coated optical ring resonator sensor relies on light-analyte interaction to convert the presence of biochemical molecules into quantitatively measurable optical signals. The resonant wavelength of light circulates along the graphene-coated ring resonator and has an evanescent field that reaches several hundred nanometers into the biochemical molecules to interact repeatedly with the analytes near the resonator surface. Graphene-coated optical ring resonators are more sensitive and can achieve high quality factors. The schematic diagram of the graphene film coated on the optical fiber end pigtail is displayed in Figure $\mathbf{4 H}$. In this approach, light is 

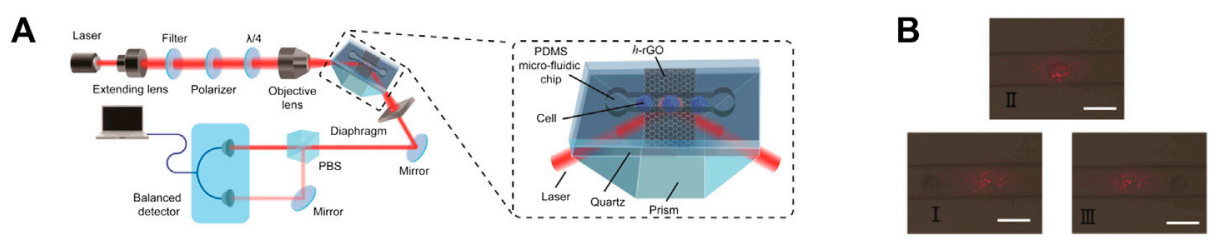

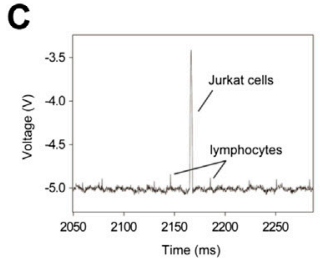

$\mathbf{F}$

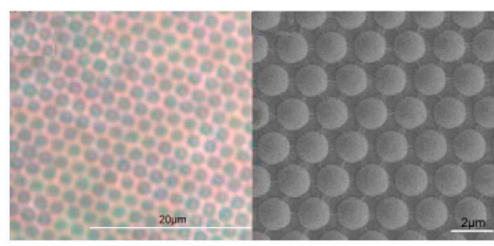

D

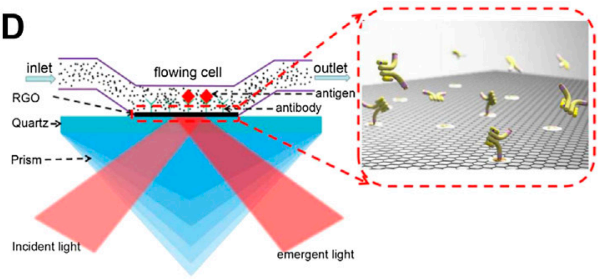

G

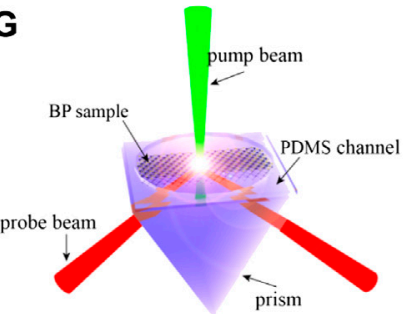

$\mathrm{E}$

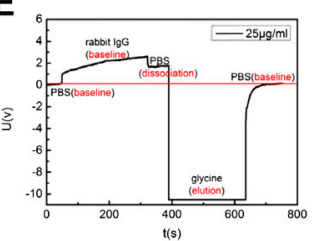

H

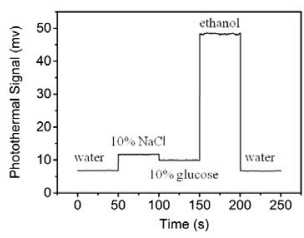

FIGURE 5 | (A) Ultrasensitive sensing of single cell using graphene-based optical sensor (Xing et al., 2014). (B) Optical images of the RGO detection window as lymphocytes roll across it. The scale bar is $15 \mu \mathrm{m}$. (C) Discrete time-dependent changes in signal that correspond to mixed lymphocytes and Jurkat cells as they roll across the detection window. (D) The RGO-based optical sensor for detecting specific protein (Jiang et al., 2017b). (E) Signal changes caused by the interaction of antigen and antibody. (F) Optical microscopy and SEM image of reduced graphene oxide microshell (RGOM) (Jiang et al., 2017a). (G) Schematic diagram of the photothermal detection experimental setup (Gao et al., 2018b). (H) Time-dependent changes in photothermal signal when different kinds of liquid medium are injected.

needed for single-ended probes with a common input and output path and works in reflection mode. In 2013, Some et al. developed novel one-headed polymer optical fiber sensor arrays using hydrophilic GO and hydrophobic RGO (Some et al., 2013). The working principle of this optical gas sensor is based on the change in the reflection of light by the interfacial layer at the fiber end facet, which in turn is induced by changes in the refractive index of the GO and RGO layers. The response of the GO- and RGO-coated sensors for volatile organic compounds, including hydrazine, nitromethane, diethylamine, ethanol, methanol, acetone, tetrahydrofuran (THF), and dichloromethane (MC), are displayed in Figure 4I. Besides, the eco-friendly physical properties of GO allow for faster sensing and higher sensitivity when compared to RGO even under extreme environments of over $90 \%$ humidity, making it the best choice for gas sensor. Although graphene-based optical fiber sensors have been widely used, there are still many difficulties to overcome in reality. It is difficult for graphene to be perfectly transferred to the surface of the optical fiber, and its thickness and quality are also difficult to control. Then, the stability and repeatability of graphene-based optical fiber sensors still need to be improved.

\section{Other Kind of Graphene-Based Optical Sensors}

The interaction between incident beam and graphene mainly includes interband and intraband transitions. In the far infrared and $\mathrm{THz}$ bands, the electronic response consists mainly of intraband transition (free carrier response), which can be well described by the Drude model. Instead, in the near infrared and visible range, the absorption of graphene consists mainly of interband transition, which is wavelength independent. Since the absorption of single layer graphene is only $2.3 \%$, a variety of structures have been designed to enhance the absorption of graphene (Mueller et al., 2010; Koppens et al., 2011; Wang et al., 2013b; Pirruccio et al., 2013; Pospischil et al., 2013).

In 2013, Ye et al. discovered that graphene exhibits strong polarization dependent optical absorption under total internal reflection (Ye et al., 2013). Compared with the limited universal absorbance of 2.3\%, a larger absorption was observed in monolayer, bilayer, and few-layer graphenes for transverse electric (TE) wave under total internal reflection. Based on the polarization-sensitive absorption effect, Wang et al. proposed a method to accurately count the number of layers for both exfoliated and chemical vapor deposition graphene on transparent substrate (Wang et al., 2013a). Their method is useful for graphene tests on transparent substrates, which is different from the commonly used $\mathrm{SiO}_{2} / \mathrm{Si}$ substrate.

In addition, the polarized absorption optical properties of graphene have also been used in sensor design. In 2014, Xing et al. used the polarization dependent optical absorption of graphene in combination with microfluidic technology to achieve high sensitivity detection of cancer cells (Xing et al., 2014). They obtained a graphene-based optical refractive index 
sensor with high resolution $\left(1.7 \times 10^{-8}\right)$ and sensitivity $(4.3 \times$ $10^{7} \mathrm{mV} / \mathrm{RIU}$ ), as well as an extensive dynamic range. Figure $5 \mathrm{~A}$ displays a schematic diagram of the graphene-based optical sensor. The probe beam is generated by a He-Ne laser with a wavelength of $632.8 \mathrm{~nm}$, and its direction of polarization is modulated by a polarizer and quarter-wave plate. The probe beam is then focused onto the center of its microfluidic channel. The inset of Figure 5A displays the schematic of the graphene-based optical single-cell sensor (GSOCS), which consists of a polydimethylsiloxane (PDMS) microfluidic chip/ h-RGO/quartz sandwich structure on the prism. After interacting with graphene, the probe beam is separated into $s$ - and $p$-polarized beams by a polarization beam splitter. The difference in intensity of the two modes is measured by a balanced detector. The researchers regarded cancer cells and normal cells as microspheres with different refractive indices. When cancer cells and normal cells pass through the surface of graphene, the refractive index changes induced are different, thus enabling the detection of cancer cells. Figure 5B illustrates the passing of cells through the microfluidic channel. In Figure 5C, the high and low voltage levels represent the signals from the cancer cell and normal cells. The GSOCS can achieve high sensitivity detection of cancer cells, which has significance for the early diagnosis and treatment of cancer. In 2017, Jiang et al. used graphene-based optical sensors to achieve high sensitivity detection of rabbit IgG (Jiang et al., 2017b). Figure 5D displays the schematic of the graphene-based optical sensor. In this process, graphene acts not only as a molecular link layer to inoculate antibodies, but also as a sensing layer to detect changes in the refractive index caused by the interaction of antigens and antibodies. Figure 5E displays a real-time measuring result of the biosensor after fabrication and biochemical treatment. The whole process of biomolecular interaction between antigen and antibody can be observed clearly, as shown in Figure 5D, which is similar to the dynamic process of biomolecular interaction of SPR-based sensor. Compared with a commercial SPR apparatus, graphene-based optical sensors can achieve higher sensitivity detection. The graphene based optical sensor shows a satisfactory response to rabbit IgG with a minimum concentration of $0.0625 \mu \mathrm{g} / \mathrm{ml}$. Furthermore, Jiang et al. developed a reduced graphene oxide microshell (RGOM)based optical biosensor for the determination of goat antirabbit IgG (Jiang et al., 2017a). In Figure 5F, the RGOM was prepared through a self-assembly of monolayer of monodisperse polystyrene microspheres. Through high temperature reduction, the RGOM was fabricated to inoculate rabbit IgG. Compared with RGO, the periodic microshells allowed for a simpler functionalization and modification of RGOM with biomolecules. This method is promising for immobilizing biomolecules on graphene surfaces and for the fabrication of biosensors with enhanced sensitivity.

In 2018, Gao et al. used the polarization dependent absorption of two-dimensional materials and a modulated pump beam to measure their photothermal signal (Gao et al., 2018a; Gao et al., 2018b; Gao et al., 2018c). Photothermal detection (PTD) is a refractive index sensing optical technique, in which a modulated pump beam is absorbed by two-dimensional materials, leading to a local change in the refractive index of the photothermal medium. The propagation of the probe beam at different wavelengths is modified by the produced periodical change of the refractive index. Figure 5G displays the schematic diagram of the PTD experimental setup. The pump beam used in the experiments is produced by a semiconductor laser at a wavelength of $532 \mathrm{~nm}$. The pump laser is linearly polarized, with its polarization angle being changed via a half-wave plate, and is regulated to a certain frequency through an acousto-optic modulator. After reflecting on the mirror, the pump beam is then focused onto the samples through the objective lens. The probe beam is generated by a $\mathrm{He}-\mathrm{Ne}$ laser at $632.8 \mathrm{~nm}$ and its polarization is modulated by the polarizer and a one-half plate. After interacting with the sample, the probe beam is split into $s$ - and $p$-polarized beams by a polarization beam splitter. The detector measures the difference between $s$ - and $p$-polarization caused by the modulated pump beam. By exploring the effect of the polarization angle of the pump beam on photothermal signal, Gao et al. explored the photothermal anisotropy of BP and $\mathrm{ReSe}_{2}$. Furthermore, the photothermal anisotropies of $\mathrm{BP} / \mathrm{ReSe}_{2}$ heterostructures were also explored. The photothermal contrasts of samples were observed to change with different stacking angles indicating that the photothermal anisotropy of heterostructures is dependent on the stacking angle. These findings provide new prospects for designing novel optical devices based on two-dimensional anisotropic materials. In addition, the PTD technique has been used for identification of the crystalline orientation of anisotropic two-dimensional materials on a transparent substrate. Compared with traditional crystal orientation determination methods, the PTD overcomes typical challenges associated with transparent substrates, including insulating and rough surfaces, and enables the unambiguous identification of crystalline orientation. Figure $\mathbf{5 H}$ displays the effect of different types of liquid media on the photothermal signal of graphene. Gao et al. proved that the thermal conductivity, heat capacity, and thermally-induced refractive index changes of the liquid medium will cause the change in the photothermal signal, indicating that the PTD technique can be implemented into a new type of optical sensor.

\section{CONCLUSION AND OUTLOOK}

In summary, the recent progress in graphene and its derivativesbased optical sensors have been reviewed, covering aspects related to fluorescence sensing, graphene-based substrates for surface-enhanced Raman scattering, optical fiber biological sensors, and other kinds of graphene based optical sensors. The last few years have witnessed a dramatic increase in research effort on graphene-based optical sensors, both at the fundamental level and from a technological point of view. Graphene and its derivatives-based optical sensors have several advantages, including fast response, high sensitivity, 
and high flexibility, and are widely used in cancer cell imaging, DNA sensing and protein detection. Although tremendous efforts have been devoted to this research field over the past several years, there still undeniably remain several significant issues that are required to be addressed and explored. The challenges involved include not only the strategies to synthesize graphene and its derivatives but also the integration of sensors into practical applications. In the synthesis process, a major challenge is to synthesize highquality graphene, and its properties can be tuned through effective chemical methods. Another challenge is to achieve a low-cost, environmentally friendly preparation method for graphene and its derivatives. For the practical application of optical sensors based on graphene and its derivatives, the primary issue is to develop high-sensitivity and highspecificity sensors, while another important objective is to enable miniaturization for integration into wearable devices. Overcoming the above challenges will not only boost graphene and its derivatives toward applications in optical sensing technology, but will also greatly advantage peoples' life.

\section{REFERENCES}

Al-Ogaidi, I., Gou, H., Aguilar, Z. P., Guo, S., Melconian, A. K., Al-Kazaz, A. K. A., et al. (2014). Detection of the ovarian cancer biomarker CA-125 using chemiluminescence resonance energy transfer to graphene quantum dots. Chem. Commun. (Camb). 50, 1344-1346. doi:10.1039/ c3cc47701k

Alvarez-Puebla, R. A., and Liz-Marzán, L. M. (2012). SERS detection of small inorganic molecules and ions. Angew. Chem. Int. Ed. 51, 11214-11223. doi:10. 1002/anie. 201204438

Ananthanarayanan, A., Wang, X., Routh, P., Sana, B., Lim, S., Kim, D.-H., et al. (2014). Facile synthesis of graphene quantum dots from 3D graphene and their application for Fe3+Sensing. Adv. Funct. Mater. 24, 3021-3026. doi:10.1002/ adfm.201303441

Avouris, P., and Dimitrakopoulos, C. (2012). Graphene: synthesis and applications. Mater. Today 15, 86-97. doi:10.1016/s1369-7021(12)70044-5

Avouris, P., and Xia, F. (2012). Graphene applications in electronics and photonics. MRS Bull. 37, 1225. doi:10.1557/mrs.2012.206

Balandin, A. A., Ghosh, S., Bao, W., Calizo, I., Teweldebrhan, D., Miao, F., et al. (2008). Superior thermal conductivity of single-layer graphene. Nano Lett. 8, 902-907. doi:10.1021/nl0731872

Bao, Q., Zhang, H., Wang, B., Ni, Z., Lim, C. H. Y. X., Wang, Y., et al. (2011). Broadband graphene polarizer. Nat. Photon. 5, 411-415. doi:10.1038/nphoton. 2011.102

Bolotin, K. I., Sikes, K. J., Jiang, Z., Klima, M., Fudenberg, G., Hone, J., et al. (2008). Ultrahigh electron mobility in suspended graphene. Solid State Commun. 146, 351-355. doi:10.1016/j.ssc.2008.02.024

Bonaccorso, F., Sun, Z., Hasan, T., and Ferrari, A. C. (2010). Graphene photonics and optoelectronics. Nat. Photon. 4, 611. doi:10.1038/nphoton.2010.186

Chen, J.-L., Yan, X.-P., Meng, K., and Wang, S.-F. (2011). Graphene oxide based photoinduced charge transfer label-free near-infrared fluorescent biosensor for dopamine. Anal. Chem. 83, 8787-8793. doi:10.1021/ac2023537

Cheng, Z., Li, Q., Li, Z., Zhou, Q., and Fang, Y. (2010). Suspended graphene sensors with improved signal and reduced noise. Nano Lett. 10, 1864-1868. doi:10. $1021 / \mathrm{nl} 100633 \mathrm{~g}$

Choi, J.-H., Kim, T.-H., El-Said, W. A., Lee, J.-H., Yang, L., Conley, B., et al. (2020). Situ detection of neurotransmitters from stem cell-derived neural interface at the single-cell level via graphene-hybrid SERS nanobiosensing. Nano Lett. 20, 7670-7679. doi:10.1021/acs.nanolett.0c03205

Cialla-May, D., Zheng, X.-S., Weber, K., and Popp, J. (2017). Recent progress in surface-enhanced Raman spectroscopy for biological and biomedical

\section{AUTHOR CONTRIBUTIONS}

$\mathrm{X}-\mathrm{GG}$ and $\mathrm{L}-\mathrm{XC}$ conceived the idea and designed the manuscript. $\mathrm{X}-\mathrm{GG}$ wrote the paper. W-SJ, X-KL, and FX participated in the collection and sorting of relevant materials. All authors discussed the results and commented on the manuscript.

\section{FUNDING}

This work was supported by the Natural Science Foundation ofChina (Grant 62005189), Natural Science Foundation of Shanxi Province (201901D211071), the open foundation of Key Laboratory of Weak Light Nonlinear Photonics, Ministry of Education (OS20-2), the Science and Technology Research Project of Henan Province (182102311130), the Key Scientific Research Items of Henan Province Colleges and Universities (20A416006), the Doctoral Research Grant Program of Xinxiang Medical University (XYBSKYZZ201719), the Natural Science Foundation of Henan Province (Grant No. 182300410107).

applications: from cells to clinics. Chem. Soc. Rev. 46, 3945-3961. doi:10. 1039/c7cs00172j

Cushing, S. K., Li, M., Huang, F., and Wu, N. (2014). Origin of strong excitation wavelength dependent fluorescence of graphene oxide. ACS Nano. 8, 1002-1013. doi: $10.1021 / \mathrm{nn} 405843 \mathrm{~d}$

Dalfovo, M. C., Lacconi, G. I., Moreno, M. N., Yappert, M. C., Sumanasekera, G. U., Salvarezza, R. C., et al. (2014). Synergy between graphene and au nanoparticles (heterojunction) towards quenching, improving Raman signal, and UV light sensing. ACS Appl. Mater. Interfaces 6, 6384-6391. doi:10.1021/am405753t

Dash, J. N., and Jha, R. (2015). On the performance of graphene-based D-shaped photonic crystal fibre biosensor using surface plasmon resonance. Plasmonics. 10, 1123-1131. doi:10.1007/s11468-015-9912-7

Dong, H., Gao, W., Yan, F., Ji, H., and Ju, H. (2010). Fluorescence resonance energy transfer between quantum dots and graphene oxide for sensing biomolecules. Anal. Chem. 82, 5511-5517. doi:10.1021/ac100852z

Dreyer, D. R., Murali, S., Zhu, Y., Ruoff, R. S., and Bielawski, C. W. (2011). Reduction of graphite oxide using alcohols. J. Mater. Chem. 21, 3443-3447. doi:10.1039/c0jm02704a

Duan, B., Zhou, J., Fang, Z., Wang, C., Wang, X., Hemond, H. F., et al. (2015). Surface enhanced Raman scattering by graphene-nanosheet-gapped plasmonic nanoparticle arrays for multiplexed DNA detection. Nanoscale 7, 12606-12613. doi:10.1039/c5nr02164b

Eda, G., and Chhowalla, M. (2010). Chemically derived graphene oxide: towards large-area thin-film electronics and optoelectronics. Adv. Mater. 22, 2392-2415. doi:10.1002/adma.200903689

Fowler, J. D., Allen, M. J., Tung, V. C., Yang, Y., Kaner, R. B., and Weiller, B. H. (2009). Practical chemical sensors from chemically derived graphene. ACS Nano. 3, 301-306. doi:10.1021/nn800593m

Frasco, M. F., and Chaniotakis, N. (2009). Semiconductor quantum dots in chemical sensors and biosensors. Sensors 9, 7266-7286. doi:10.3390/s90907266

Galande, C., Mohite, A. D., Naumov, A. V., Gao, W., Ci, L., Ajayan, A., et al. (2011). Quasi-molecular fluorescence from graphene oxide. Sci. Rep. 1, 1-5. doi:10. 1038/srep00085

Gan, S., Cheng, C., Zhan, Y., Huang, B., Gan, X., Li, S., et al. (2015). A highly efficient thermo-optic microring modulator assisted by graphene. Nanoscale 7, 20249-20255. doi:10.1039/c5nr05084g

Gao, X.-G., Chen, G.-X., Li, D.-K., Li, X.-K., Liu, Z.-B., and Tian, J.-G. (2018a) Modulation of photothermal anisotropy using black phosphorus/rhenium diselenide heterostructures. Nanoscale 10, 10844-10849. doi:10.1039/ c8nr02229a

Gao, X., Chen, G., Li, D., Li, X., Liu, Z., and Tian, J. (2018b). High-accuracy measurement of the crystalline orientation of anisotropic two-dimensional 
materials using photothermal detection. J. Mater. Chem. C. 6, 5849-5856. doi:10.1039/c8tc01822g

Gao, X. G., Jiang, W. S., Chen, G. X., Li, X. K., Li, D. K., Huang, K. X., et al. (2018c). Visualizing photothermal anisotropy in black phosphorus by total internal reflection pump-probe technique. Adv. Mater. Interfaces 5, 1701605. doi:10. 1002/admi.201701605

Gao, X.-G., Li, X.-K., Xin, W., Chen, X.-D., Liu, Z.-B., and Tian, J.-G. (2020). Fabrication, optical properties, and applications of twisted two-dimensional materials. Nanophotonics. 9, 1717-1742. doi:10.1515/nanoph-2020-0024

Gilje, S., Han, S., Wang, M., Wang, K. L., and Kaner, R. B. (2007). A chemical route to graphene for device applications. Nano Lett. 7, 3394-3398. doi:10.1021/ nl0717715

Han, J., Ryu, S., Sohn, D., and Im, S. (2014). Mechanical strength characteristics of asymmetric tilt grain boundaries in graphene. Carbon 68, 250-257. doi:10. 1016/j.carbon.2013.10.085

Hao, Q., Wang, B., Bossard, J. A., Kiraly, B., Zeng, Y., Chiang, I.-K., et al. (2012). Surface-enhanced Raman scattering study on graphene-coated metallic nanostructure substrates. J. Phys. Chem. C 116, 7249-7254. doi:10.1021/ jp209821g

He, L., Li, J., and Xin, J. H. (2015). A novel graphene oxide-based fluorescent nanosensor for selective detection of $\mathrm{Fe}^{3+}$ with a wide linear concentration and its application in logic gate. Biosen. Bioelectron. 70, 69-73. doi:10.1016/j.bios. 2015.01.075

He, Y., and Cui, H. (2012). Fabrication of luminol and lucigenin bifunctionalized gold nnanoparticles/graphene oxide nanocomposites with dual-wavelength chemiluminescence. J. Phys. Chem. C. 116, 12953-12957. doi:10.1021/ jp303304z

Huang, H., Li, Z., She, J., and Wang, W. (2012). Oxygen density dependent band gap of reduced graphene oxide. J. Appl. Phys. 111, 054317. doi:10.1063/1.3694665

Huang, X., Yin, Z., Wu, S., Qi, X., He, Q., Zhang, Q., et al. (2011). Graphene-based materials: synthesis, characterization, properties, and applications. Small 7, 1876-1902. doi:10.1002/smll.201002009

Ilkhani, H., Hughes, T., Li, J., Zhong, C. J., and Hepel, M. (2016). Nanostructured SERS-electrochemical biosensors for testing of anticancer drug interactions with DNA. Biosen. Bioelectron. 80, 257-264. doi:10.1016/j.bios.2016.01.068

Iranifam, M., Babakalak, P. R., Imani-Nabiyyi, A., Abolghasemi, M. M., and Khataee, A. (2016). Graphene oxide induced chemiluminescence used for quenchometric determination of dobutamine hydrochloride. Anal. Methods 8, 3496-3502. doi:10.1039/c6ay00363j

Jiang, W.-S., Xin, W., Chen, S.-N., Li, C.-B., Gao, X.-G., Pan, L.-T., et al. (2017a). Microshell arrays enhanced sensitivity in detection of specific antibody for reduced graphene oxide optical sensor. Sensors. 17, 221. doi:10.3390/ s17020221

Jiang, W.-S., Xin, W., Xun, S., Chen, S.-N., Gao, X.-G., Liu, Z.-B., et al. (2017b). Reduced graphene oxide-based optical sensor for detecting specific protein. Sens. Actuators B Chem. 249, 142-148. doi:10.1016/j.snb.2017.03.175

Jung, J. H., Cheon, D. S., Liu, F., Lee, K. B., and Seo, T. S. (2010). A graphene oxide based immuno-biosensor for pathogen detection. Angew. Chem. 122, 5844-5847. doi:10.1002/anie.201001428

Kalluru, P., Vankayala, R., Chiang, C.-S., and Hwang, K. C. (2016). Nano-graphene oxide-mediated in vivo fluorescence imaging and bimodal photodynamic and photothermal destruction of tumors. Biomaterials 95, 1-10. doi:10.1016/j. biomaterials.2016.04.006

Kang, T., Yoo, S. M., Yoon, I., Lee, S. Y., and Kim, B. (2010). Patterned multiplex pathogen DNA detection by Au particle-on-wire SERS sensor. Nano Lett. 10, 1189-1193. doi:10.1021/nl1000086

Kim, J. A., Hwang, T., Dugasani, S. R., Amin, R., Kulkarni, A., Park, S. H., et al. (2013). Graphene based fiber optic surface plasmon resonance for bio-chemical sensor applications. Sens. Actuators B Chem. 187, 426-433. doi:10.1016/j.snb. 2013.01.040

Kim, J. T., and Choi, C.-G. (2012). Graphene-based polymer waveguide polarizer. Opt. Express 20, 3556-3562. doi:10.1364/OE.20.003556

Kneipp, K., Wang, Y., Kneipp, H., Perelman, L. T., Itzkan, I., Dasari, R. R., et al. (1997). Single molecule detection using surface-enhanced Raman scattering (SERS). Phys. Rev. Lett. 78, 1667. doi:10.1103/physrevlett.78.1667

Kong, J., Chapline, M. G., and Dai, H. (2001). Functionalized carbon nanotubes for molecular hydrogen sensors. Adv. Mater. 13, 1384-1386. doi:10.1002/15214095(200109)13:18<1384::aid-adma1384>3.0.co;2-8
Koppens, F. H., Chang, D. E., and Garcia De Abajo, F. J. (2011). Graphene plasmonics: a platform for strong light-matter interactions. Nano Lett. 11, 3370-3377. doi:10.1021/nl201771h

Kuang, H., Chen, W., Yan, W., Xu, L., Zhu, Y., Liu, L., et al. (2011). Crown ether assembly of gold nanoparticles: melamine sensor. Biosen. Bioelectron. 26, 2032-2037. doi:10.1016/j.bios.2010.08.081

Kwak, S. Y., Yang, J. K., Jeon, S. J., Kim, H. I., Yim, J., Kang, H., et al. (2014). Luminescent graphene oxide with a peptide-quencher complex for optical detection of cell-secreted proteases by a turn-on response. Adv. Funct. Mater. 24, 5119-5128. doi:10.1002/adfm.201400001

Lai, H., Xu, F., Zhang, Y., and Wang, L. (2018). Recent progress on graphene-based substrates for surface-enhanced Raman scattering applications. J. Mater. Chem. B 6, 4008-4028. doi:10.1039/c8tb00902c

Landau, L. D. (1937). Zur Theorie der phasenumwandlungen II. Phys. Z. Sowjetunion 11, 26-35.

Leem, J., Wang, M. C., Kang, P., and Nam, S. (2015). Mechanically self-assembled, three-dimensional graphene-gold hybrid nanostructures for advanced nanoplasmonic sensors. Nano Lett. 15, 7684-7690. doi:10.1021/acs.nanolett. $5 \mathrm{~b} 03672$

Li, C., Liu, Q., Peng, X., and Fan, S. (2015). Analyzing the temperature sensitivity of Fabry-Perot sensor using multilayer graphene diaphragm. Opt. Express 23, 27494-27502. doi:10.1364/OE.23.027494

Li, J., Chen, L., Lou, T., and Wang, Y. (2011). Highly sensitive SERS detection of $\mathrm{As}^{3+}$ ions in aqueous media using glutathione functionalized silver nanoparticles. ACS Appl. Mater. Interfaces 3, 3936-3941. doi:10.1021/am200810x

Li, M., Cushing, S. K., Zhou, X., Guo, S., and Wu, N. (2012). Fingerprinting photoluminescence of functional groups in graphene oxide. J. Mater. Chem. 22, 23374-23379. doi:10.1039/c2jm35417a

Li, M., Zhou, X., Ding, W., Guo, S., and Wu, N. (2013a). Fluorescent aptamerfunctionalized graphene oxide biosensor for label-free detection of mercury (II). Biosen. Bioelectron. 41, 889-893. doi:10.1016/j.bios.2012.09.060

Li, Y.-T., Qu, L.-L., Li, D.-W., Song, Q.-X., Fathi, F., and Long, Y.-T. (2013b). Rapid and sensitive in-situ detection of polar antibiotics in water using a disposable Ag-graphene sensor based on electrophoretic preconcentration and surfaceenhanced Raman spectroscopy. Biosen. Bioelectron. 43, 94-100. doi:10.1016/j. bios.2012.12.005

Lin, D., Qin, T., Wang, Y., Sun, X., and Chen, L. (2014). Graphene oxide wrapped SERS tags: multifunctional platforms toward optical labeling, photothermal ablation of bacteria, and the monitoring of killing effect. ACS Appl. Mater. Interfaces 6, 1320-1329. doi:10.1021/am405396k

Lin, J., Shang, Y., Li, X., Yu, J., Wang, X., and Guo, L. (2017). Ultrasensitive SERS detection by defect engineering on single $\mathrm{Cu}_{2} \mathrm{O}$ superstructure particle. $A d v$. Mater. 29, 1604797. doi:10.1002/adma.201604797

Ling, X., Xie, L., Fang, Y., Xu, H., Zhang, H., Kong, J., et al. (2010). Can graphene be used as a substrate for Raman enhancement? Nano Lett. 10, 553-561. doi:10. $1021 / \mathrm{nl} 903414 \mathrm{x}$

Lipatov, A., Varezhnikov, A., Wilson, P., Sysoev, V., Kolmakov, A., and Sinitskii, A. (2013). Highly selective gas sensor arrays based on thermally reduced graphene oxide. Nanoscale 5, 5426-5434. doi:10.1039/c3nr00747b

Liu, C.-H., Chang, Y.-C., Norris, T. B., and Zhong, Z. (2014). Graphene photodetectors with ultra-broadband and high responsivity at room temperature. Nat. Nanotechnol. 9, 273-278. doi:10.1038/nnano.2014.31

Liu, Y., Dong, X., and Chen, P. (2012). Biological and chemical sensors based on graphene materials. Chem. Soc. Rev. 41, 2283-2307. doi:10.1039/c1cs15270j

Liu, Z., Li, S., Hu, C., Zhang, W., Zhong, H., and Guo, Z. (2013). pH-dependent surface-enhanced Raman scattering of aromatic molecules on graphene oxide. J. Raman Spectrosc. 44, 75-80. doi:10.1002/jrs.4142

Loh, K. P., Bao, Q., Eda, G., and Chhowalla, M. (2010). Graphene oxide as a chemically tunable platform for optical applications. Nat. Chem. 2, 1015. doi:10. 1038/nchem.907

Mak, K. F., Ju, L., Wang, F., and Heinz, T. F. (2012). Optical spectroscopy of graphene: from the far infrared to the ultraviolet. Solid State Commun. 152, 1341-1349. doi:10.1016/j.ssc.2012.04.064

Mathkar, A., Tozier, D., Cox, P., Ong, P., Galande, C., Balakrishnan, K., et al. (2012). Controlled, stepwise reduction and band gap manipulation of graphene oxide. J. Phys. Chem. Lett. 3, 986-991. doi:10.1021/jz300096t

Matsui, J., Akamatsu, K., Hara, N., Miyoshi, D., Nawafune, H., Tamaki, K., et al. (2005). SPR sensor chip for detection of small molecules using molecularly 
imprinted polymer with embedded gold nanoparticles. Anal. Chem. 77, 4282-4285. doi:10.1021/ac050227i

Modi, A., Koratkar, N., Lass, E., Wei, B., and Ajayan, P. M. (2003). Miniaturized gas ionization sensors using carbon nanotubes. Nature 424, 171-174. doi:10.1038/ nature 01777

Mueller, T., Xia, F., and Avouris, P. (2010). Graphene photodetectors for highspeed optical communications. Nat. Photon. 4, 297-301. doi:10.1038/nnano. 2009.292

Nair, R. R., Blake, P., Grigorenko, A. N., Novoselov, K. S., Booth, T. J., Stauber, T., et al. (2008). Fine structure constant defines visual transparency of graphene. Science. 320, 1308. doi:10.1126/science.1156965

Neto, A. C., Guinea, F., Peres, N. M., Novoselov, K. S., and Geim, A. K. (2009). The electronic properties of graphene. Rev. Mod. Phys. 81, 109. doi:10.1103/ RevModPhys.81.109

Novoselov, K. S., Fal, V., Colombo, L., Gellert, P., Schwab, M., and Kim, K. (2012). A roadmap for graphene. Nature 490, 192-200. doi:10.1038/nature11458

Novoselov, K. S., and Geim, A. (2007). The rise of graphene. Nat. Mater. 6, 183-191. doi:10.1038/nmat1849

Novoselov, K. S., Geim, A. K., Morozov, S. V., Jiang, D., Zhang, Y., Dubonos, S. V., et al. (2004). Electric field effect in atomically thin carbon films. Science 306, 666-669. doi:10.1126/science.1102896

Ouyang, L., Hu, Y., Zhu, L., Cheng, G. J., and Irudayaraj, J. (2017). A reusable laser wrapped graphene-Ag array based SERS sensor for trace detection of genomic DNA methylation. Biosen. Bioelectron. 92, 755-762. doi:10.1016/j.bios.2016. 09.072

Peierls, R. (1935). Quelques propriétés typiques des corps solides. Annales de l'institut Henri Poincaré 5, 177-222.

Pirruccio, G., Martin Moreno, L., Lozano, G., and GóMez Rivas, J. (2013). Coherent and broadband enhanced optical absorption in graphene. ACS Nano 7, 4810-4817. doi:10.1021/nn4012253

Pospischil, A., Humer, M., Furchi, M. M., Bachmann, D., Guider, R., Fromherz, T., et al. (2013). CMOS-compatible graphene photodetector covering all optical communication bands. Nat. Photon. 7, 892-896. doi:10.1038/ nphoton.2013.240

Pramanik, A., Chavva, S. R., Fan, Z., Sinha, S. S., Nellore, B. P. V., and Ray, P. C. (2014a). Extremely high two-photon absorbing graphene oxide for imaging of tumor cells in the second biological window. J. Phys. Chem. Lett. 5, 2150-2154. doi:10.1021/jz5009856

Pramanik, A., Fan, Z., Chavva, S. R., Sinha, S. S., and Ray, P. C. (2014b). Highly efficient and excitation tunable two-photon luminescence platform for targeted multi-color MDRB imaging using graphene oxide. Sci. Rep. 4, 6090. doi:10. 1038/srep06090

Qiang, W., Li, W., Li, X., Chen, X., and Xu, D. (2014). Bioinspired polydopamine nanospheres: a superquencher for fluorescence sensing of biomolecules. Chem. Sci. 5, 3018-3024. doi:10.1039/c4sc00085d

Robinson, J. T., Perkins, F. K., Snow, E. S., Wei, Z., and Sheehan, P. E. (2008). Reduced graphene oxide molecular sensors. Nano Lett. 8, 3137-3140. doi:10. $1021 / \mathrm{nl} 8013007$

Ryoo, S.-R., Lee, J., Yeo, J., Na, H.-K., Kim, Y.-K., Jang, H., et al. (2013). Quantitative and multiplexed microRNA sensing in living cells based on peptide nucleic acid and nano graphene oxide (PANGO). ACS Nano 7, 5882-5891. doi:10.1021/nn401183s

Sansone, L., Malachovska, V., La Manna, P., Musto, P., Borriello, A., De Luca, G., et al. (2014). Nanochemical fabrication of a graphene oxide-based nanohybrid for label-free optical sensing with fiber optics. Sens. Actuators B Chem. 202, 523-526. doi:10.1016/j.snb.2014.05.067

Sensale-Rodriguez, B., Yan, R., Kelly, M. M., Fang, T., Tahy, K., Hwang, W. S., et al. (2012). Broadband graphene terahertz modulators enabled by intraband transitions. Nat. Commun. 3, 1-7. doi:10.1038/ncomms1787

Shen, J., Zhu, Y., Yang, X., and Li, C. (2012). Graphene quantum dots: emergent nanolights for bioimaging, sensors, catalysis and photovoltaic devices. Chem. Commun. 48, 3686-3699. doi:10.1039/c2cc00110a

Shi, Y., Pramanik, A., Tchounwou, C., Pedraza, F., Crouch, R. A., Chavva, S. R., et al. (2015). Multifunctional biocompatible graphene oxide quantum dots decorated magnetic nanoplatform for efficient capture and two-photon imaging of rare tumor cells. ACS Appl. Mater. Interfaces 7, 10935-10943. doi:10.1021/acsami.5b02199
Shivananju, B. N., Yu, W., Liu, Y., Zhang, Y., Lin, B., Li, S., et al. (2017). The roadmap of graphene-based optical biochemical sensors. Adv. Funct. Mater. 27, 1603918. doi:10.1002/adfm.201603918

So, P. T., Dong, C. Y., Masters, B. R., and Berland, K. M. (2000). Two-photon excitation fluorescence microscopy. Annu. Rev. Biomed. Eng. 2, 399-429. doi:10.1146/annurev.bioeng.2.1.399

Some, S., Xu, Y., Kim, Y., Yoon, Y., Qin, H., Kulkarni, A., et al. (2013). Highly sensitive and selective gas sensor using hydrophilic and hydrophobic graphenes. Sci. Rep. 3, 1868. doi:10.1038/srep01868

Son, D. I., Kwon, B. W., Park, D. H., Seo, W.-S., Yi, Y., Angadi, B., et al. (2012). Emissive $\mathrm{ZnO}$-graphene quantum dots for white-light-emitting diodes. Nat. Nanotechnol. 7, 465-471. doi:10.1038/nnano.2012.71

Song, X., Li, S., Guo, H., You, W., Shang, X., Li, R., et al. (2019). Graphene-oxidemodified lanthanide nanoprobes for tumor-targeted visible/NIR-II luminescence imaging. Angew. Chem. Int. Ed. 58, 18981-18986. doi:10.1002/ anie. 201909416

Sun, H., Wu, L., Wei, W., and Qu, X. (2013). Recent advances in graphene quantum dots for sensing. Mater. Today 16, 433-442. doi:10.1016/j.mattod.2013.10.020

Sun, Z., Hasan, T., Torrisi, F., Popa, D., Privitera, G., Wang, F., et al. (2010a). Graphene mode-locked ultrafast laser. ACS Nano 4, 803-810. doi:10.1021/ nn901703e

Sun, Z., Popa, D., Hasan, T., Torrisi, F., Wang, F., Kelleher, E. J., et al. (2010b). A stable, wideband tunable, near transform-limited, graphene-mode-locked, ultrafast laser. Nano Res. 3, 653-660. doi:10.1007/s12274-010-0026-4

Tchounwou, C., Sinha, S. S., Viraka Nellore, B. P., Pramanik, A., Kanchanapally, R., Jones, S., et al. (2015). Hybrid theranostic platform for second near-IR window light triggered selective two-photon imaging and photothermal killing of targeted melanoma cells. ACS Appl. Mater. Interfaces 7, 20649-20656. doi:10.1021/acsami.5b05225

Tsai, M.-F., Chang, S.-H. G., Cheng, F.-Y., Shanmugam, V., Cheng, Y.-S., Su, C.-H., et al. (2013). Au nanorod design as light-absorber in the first and second biological near-infrared windows for in vivo photothermal therapy. ACS Nano 7, 5330-5342. doi:10.1021/nn401187c

Wang, F., Gu, Z., Lei, W., Wang, W., Xia, X., and Hao, Q. (2014). Graphene quantum dots as a fluorescent sensing platform for highly efficient detection of copper (II) ions. Sens. Actuators B Chem. 190, 516-522. doi:10.1016/j.snb.2013. 09.009

Wang, D.-Y., Wang, D.-W., Chen, H.-A., Chen, T.-R., Li, S.-S., Yeh, Y.-C., et al. (2015). Photoluminescence quenching of graphene oxide by metal ions in aqueous media. Carbon 82, 24-30. doi:10.1016/j.carbon.2014.10.017

Wang, L., Zheng, J., Yang, S., Wu, C., Liu, C., Xiao, Y., et al. (2015b). Two-photon sensing and imaging of endogenous biological cyanide in plant tissues using graphene quantum dot/gold nanoparticle conjugate. ACS Appl. Mater. Interfaces 7, 19509-19515. doi:10.1021/acsami.5b06352

Wang, N., Lin, M., Dai, H., and Ma, H. (2016). Functionalized gold nanoparticles/ reduced graphene oxide nanocomposites for ultrasensitive electrochemical sensing of mercury ions based on thymine-mercury-thymine structure. Biosen. Bioelectron. 79, 320-326. doi:10.1016/j.bios.2015.12.056

Wang, P., Liu, Z.-B., Chen, X.-D., Xing, F., Jiang, W.-S., Dong, B., et al. (2013a). Accurate layers determination of graphene on transparent substrate based on polarization-sensitive absorption effect. Appl. Phys. Lett. 103, 181902. doi:10. $1063 / 1.4827812$

Wang, X., Cheng, Z., Xu, K., Tsang, H. K., and Xu, J.-B. (2013b). High-responsivity graphene/silicon-heterostructure waveguide photodetectors. Nat. Photon. 7 , 888-891. doi:10.1038/nphoton.2013.241

Wu, J., Agrawal, M., Becerril, H. A., Bao, Z., Liu, Z., Chen, Y., et al. (2010). Organic light-emitting diodes on solution-processed graphene transparent electrodes. ACS Nano 4, 43-48. doi:10.1021/nn900728d

Xing, F., Meng, G.-X., Zhang, Q., Pan, L.-T., Wang, P., Liu, Z.-B., et al. (2014). Ultrasensitive flow sensing of a single cell using graphene-based optical sensors. Nano Lett. 14, 3563-3569. doi:10.1021/nl5012036

Xu, S., Jiang, S., Hu, G., Wei, J., Wang, L., Zhang, J., et al. (2015). Highly ordered graphene-isolated silver nanodot arrays as SERS substrate for detection of urinary nucleosides. Laser Phys. 25, 115601. doi:10.1088/1054-660x/25/11/ 115601

Yang, K., Feng, L., Shi, X., and Liu, Z. (2013). Nano-graphene in biomedicine: theranostic applications. Chem. Soc. Rev. 42, 530-547. doi:10.1039/c2cs35342c 
Yao, B., Wu, Y., Cheng, Y., Zhang, A., Gong, Y., Rao, Y.-J., et al. (2014a). All-optical Mach-Zehnder interferometric NH3 gas sensor based on graphene/microfiber hybrid waveguide. Sens. Actuators B Chem. 194, 142-148. doi:10.1016/j.snb. 2013.12.085

Yao, B., Wu, Y., Zhang, A., Rao, Y., Wang, Z., Cheng, Y., et al. (2014b). Graphene enhanced evanescent field in microfiber multimode interferometer for highly sensitive gas sensing. Opt. Express 22, 28154-28162. doi:10.1364/OE.22. 028154

Ye, Q., Wang, J., Liu, Z., Deng, Z.-C., Kong, X.-T., Xing, F., et al. (2013). Polarization-dependent optical absorption of graphene under total internal reflection. Appl. Phys. Lett. 102, 021912. doi:10.1063/1.4776694

Yin, F., Wu, S., Wang, Y., Wu, L., Yuan, P., and Wang, X. (2016). Self-assembly of mildly reduced graphene oxide monolayer for enhanced Raman scattering. J. Solid State Chem. 237, 57-63. doi:10.1016/j.jssc.2016.01.015

Yuan, W., and Shi, G. (2013). Graphene-based gas sensors. J. Mater. Chem. 1, 10078-10091. doi:10.1039/c3ta11774j

Zeng, F., Xu, D., Zhan, C., Liang, C., Zhao, W., Zhang, J., et al. (2018). Surfactantfree synthesis of graphene oxide coated silver nanoparticles for sers biosensing and intracellular drug delivery. ACS Appl. Nano Mater. 1, 2748-2753. doi:10. 1021/acsanm.8b00444

Zeng, S., Sreekanth, K. V., Shang, J., Yu, T., Chen, C. K., Yin, F., et al. (2015). Graphene-gold metasurface architectures for ultrasensitive plasmonic biosensing. Adv. Mater. 27, 6163-6169. doi:10.1002/adma.201501754

Zhang, J., Liao, G., Jin, S., Cao, D., Wei, Q., Lu, H., et al. (2014). All-fiber-optic temperature sensor based on reduced graphene oxide. Laser Phys. Lett. 11, 035901. doi:10.1088/1612-2011/11/3/035901

Zhang, X., Dai, Z., Si, S., Zhang, X., Wu, W., Deng, H., et al. (2017). Ultrasensitive SERS substrate integrated with uniform subnanometer scale "hot spots" created by a graphene spacer for the detection of mercury ions. Small 13, 1603347. doi:10.1002/smll.201603347

Zhang, Y., Qian, J., Wang, D., Wang, Y., and He, S. (2013). Multifunctional gold nanorods with ultrahigh stability and tunability for in vivo fluorescence imaging, SERS detection, and photodynamic therapy. Angew. Chem. Int. Ed. 52, 1148-1151. doi:10.1002/anie.201207909
Zhang, Z., Liu, Q., Gao, D., Luo, D., Niu, Y., Yang, J., et al. (2015). Graphene oxide as a multifunctional platform for Raman and fluorescence imaging of cells. Small 11, 3000-3005. doi:10.1002/smll.201403459

Zhao, Y., Li, X., Liu, Y., Zhang, L., Wang, F., and Lu, Y. (2017). High performance surface-enhanced Raman scattering sensing based on $\mathrm{Au}$ nanoparticlemonolayer graphene-Ag nanostar array hybrid system. Sens. Actuators B Chem. 247, 850-857. doi:10.1016/j.snb.2017.03.063

Zheng, P., and Wu, N. (2017). Fluorescence and sensing applications of graphene oxide and graphene quantum dots: a review. Chem. Asian J. 12, 2343-2353. doi:10.1002/asia.201700814

Zhu, H., Xu, H., Yan, Y., Zhang, K., Yu, T., Jiang, H., et al. (2014). Highly fluorescent graphene oxide as a facile and novel sensor for the determination of hypochlorous acid. Sens. Actuators B Chem. 202, 667-673. doi:10.1016/j.snb.2014.06.002

Zhu, S., Zhang, J., Qiao, C., Tang, S., Li, Y., Yuan, W., et al. (2011). Strongly greenphotoluminescent graphene quantum dots for bioimaging applications. Chem. Commun. 47, 6858-6860. doi:10.1039/c1cc11122a

Zhu, X., Shi, L., Schmidt, M. S., Boisen, A., Hansen, O., Zi, J., et al. (2013). Enhanced light-matter interactions in graphene-covered gold nanovoid arrays. Nano Lett. 13, 4690-4696. doi:10.1021/nl402120t

Zhu, Y., Murali, S., Cai, W., Li, X., Suk, J. W., Potts, J. R., et al. (2010). Graphene and graphene oxide: synthesis, properties, and applications. Adv. Mater. 22, 3906-3924. doi:10.1002/adma.201001068

Conflict of Interest: The authors declare that the research was conducted in the absence of any commercial or financial relationships that could be construed as a potential conflict of interest.

Copyright (c) 2021 Gao, Cheng, Jiang, Li and Xing. This is an open-access article distributed under the terms of the Creative Commons Attribution License (CC BY) The use, distribution or reproduction in other forums is permitted, provided the original author(s) and the copyright owner(s) are credited and that the original publication in this journal is cited, in accordance with accepted academic practice. No use, distribution or reproduction is permitted which does not comply with these terms. 